\title{
Impact of the COVID-19 Pandemic on Pediatric Cystic Fibrosis Pulmonary Exacerbations
}

\author{
Shreya Patel ${ }^{1}$, Misty Thompson ${ }^{1}$, James Slaven ${ }^{2}$, Don Sanders ${ }^{1}$, and Clement Ren ${ }^{3}$ \\ ${ }^{1}$ Indiana University School of Medicine \\ ${ }^{2}$ Indiana University Purdue University at Indianapolis \\ ${ }^{3}$ Indiana University System
}

November 9, 2020

\author{
Abstract \\ To assess the impact of COVID-19 restrictions on cystic fibrosis (CF) pulmonary exacerbations (PEx) we performed a retro- \\ spective review of PEx events at our CF Center and compared the rate of PEx in 2019 vs 2020. Restrictions on social interaction \\ due to the COVID-19 pandemic were associated with a lower rate of PEx at our pediatric CF Center, suggesting that these \\ restrictions also reduced exposure to other respiratory viral infection in children with $\mathrm{CF}$. \\ Impact of the COVID-19 Pandemic on Pediatric Cystic Fibrosis Pulmonary Exacerbations \\ Shreya Patel BS ${ }^{1}$, Misty D Thompson $\mathrm{BS}^{2,3}$, James E. Slaven $\mathrm{MS}^{4}$, Don B. Sanders MD MS ${ }^{2,3}$, and Clement \\ L. Ren MD MBA ${ }^{2,3}$ \\ ${ }^{1}$ Indiana University School of Medicine, Indianapolis, IN USA \\ ${ }^{2}$ Division of Pediatric Pulmonology, Allergy, and Sleep Medicine, Riley Hospital for Children, Indianapolis, \\ IN USA \\ ${ }^{3}$ Department of Pediatrics, Indiana University School of Medicine, Indianapolis, IN USA \\ ${ }^{4}$ Department of Biostatistics, Indiana University/Purdue University at Indianapolis, IN USA \\ Correspondence to: \\ Clement L. Ren, MD, MBA \\ Division of Pediatric Pulmonology, Allergy, and Sleep Medicine \\ Riley Hospital for Children \\ ROC 4270
}

705 Riley Hospital Drive

Indianapolis, IN 46202

Phone: 317-948-7180

FAX: 317-944-7247

Email: clren@iu.edu

Keywords: cystic fibrosis, exacerbation, COVID-19, epidemiology 
Acknowledgements: S. Patel was supported by NIH T35HL110854.

Abstract:

To assess the impact of COVID-19 restrictions on cystic fibrosis (CF) pulmonary exacerbations (PEx) we performed a retrospective review of PEx events at our CF Center and compared the rate of PEx in 2019 vs 2020. Restrictions on social interaction due to the COVID-19 pandemic were associated with a lower rate of PEx at our pediatric CF Center, suggesting that these restrictions also reduced exposure to other respiratory viral infection in children with $\mathrm{CF}$.

To The Editor:

The COVID-19 pandemic resulted in widespread restrictions on social interactions and lockdowns in many areas of the world [1]. The impact of these measures on cystic fibrosis (CF) pulmonary exacerbations (PEx) has not been described. Respiratory viral infections are a common trigger for PEx [2,3], and COVID-19 restrictions might lead to fewer PEx by reducing exposure to viruses in general, not just SARS-CoV2. To test the hypothesis that the COVID-19 lockdown would be associated with a lower PEx incidence we compared PEx data at our CF Center from the time with the most stringent restrictions in Indiana to the same time interval in 2019.

We identified PEx events by performing a retrospective chart review of children with $\mathrm{CF}$ followed at the CF Center at Riley Hospital for Children. We limited the age range to 2-11 years to avoid any potential confounding by elexacaftor/tezacaftor/ivacaftor therapy $[4,5]$. We collected data from two time intervals in both 2019 and 2020: January 1 to March 15 (which corresponded to the months prior to the COVID-19 detection in Indiana) and March 16 to May 15 (which corresponded with the months of peak COVID-19 incidence in 2020). PEx was determined by the treating clinician based on changes in signs, symptoms, and/or lung function. Every PEx event was analyzed during these timeframes, and data collected included demographics, height and weight, lung function, microbiology, location of encounter, and antibiotic treatment. Data were analyzed using descriptive statistics and generalized linear models where data had repeated measures. This project was reviewed and approved by our local institutional review board prior to data collection.

The number and location of PEx events during the time intervals studies are shown in the Table. The number of PEx events were significantly decreased in both time intervals in 2020 compared to 2019, although the decline was even greater from March 16 to May 15. The location of patients' PEx events also differed between 2019 and 2020, with a significantly lower percentage of PEx occurring through in-person clinic visits and a much larger percentage occurring through phone encounters. The number of patients (80 in 2019 and 78 in 2020) and their clinical characteristics, such as sex, genotype, respiratory culture history, baseline lung function, and nutritional indices were similar for both years, suggesting that our observations were not due to an overall improvement in the health of our patients. However, whereas $100 \%$ of clinic visits in 2019 and from January 1-March 162020 were in-person, only 16\% of visits during the March 16-May 152020 time interval were in-person.

Our analysis shows that COVID-19 restrictions were associated with decreased PEx events and a shift towards PEx diagnosis through phone encounters rather than in-person visits. We speculate that this may have occurred due to reduced exposure to respiratory viral infections in general. It is also possible that because our patients and their parents were both at home more, there was improved adherence. We also observed a reduction in PEx rate prior to March 16, which may reflect the fact that patients were already engaging in a degree of self-isolation prior to the statewide lockdown. The decrease in in-person PEx events reflects the increased use of telehealth at the height of the COVID-19 pandemic in Indiana. Limitations of our analysis include the retrospective study design and the fact that we only analyzed data from a single CF Center.

In summary, restrictions on social interaction due to the COVID-19 pandemic were associated with a lower rate of PEx at our pediatric CF Center. These results are consistent with other studies demonstrating the importance of respiratory viral infections as a trigger for PEx and will help CF clinician anticipate the 
burden of CF lung disease should severe lockdowns due to COVID-19 occur again in their region.

References:

1. Studdert DM, and Hall MA. Disease Control, Civil Liberties, and Mass Testing - Calibrating Restrictions during the Covid-19 Pandemic. New Engl J Med. 2020;383:102-4.

2. Meyer VMC, Siqueira MM, Costa P, Caetano BC, Oliveira Lopes JC, Folescu TW, et al. Clinical impact of respiratory virus in pulmonary exacerbations of children with Cystic Fibrosis. PLoS One. 2020;15:e0240452.

3. Wat D, Gelder C, Hibbitts S, Cafferty F, Bowler I, Pierrepoint M, et al. The role of respiratory viruses in cystic fibrosis. J Cyst Fibros. 2008;7:320-8.

4. Heijerman HGM, McKone EF, Downey DG, Van Braeckel E, Rowe SM, Tullis E, et al. Efficacy and safety of the elexacaftor plus tezacaftor plus ivacaftor combination regimen in people with cystic fibrosis homozygous for the F508del mutation: a double-blind, randomised, phase 3 trial. Lancet. 2019;394:1940-8.

5. Middleton PG, Mall MA, Drevinek P, Lands LC, McKone EF, Polineni D, et al. Elexacaftor-TezacaftorIvacaftor for Cystic Fibrosis with a Single Phe508del Allele. N Engl J Med. 2019;381:1809-19.

Hosted file

Shreya Table.pdf available at https://authorea.com/users/374130/articles/491738-impact-ofthe-covid-19-pandemic-on-pediatric-cystic-fibrosis-pulmonary-exacerbations 